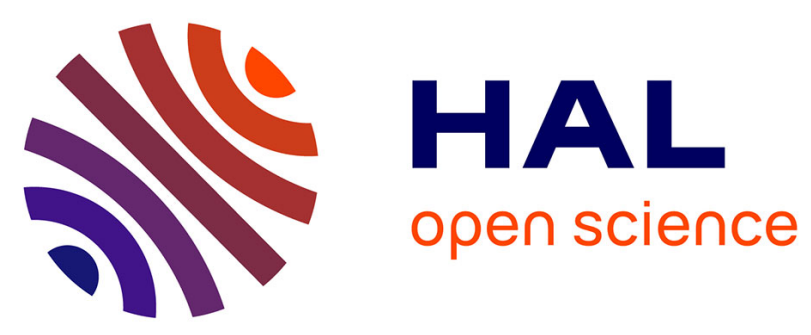

\title{
Competing Dispersive Interactions: From Small Energy Differences to Large Structural Effects in Methyl Jasmonate and Zingerone
}

Iciar Uriarte, Aran Insausti, Emilio Cocinero, Atef Jabri, Isabelle Kleiner, Halima Mouhib, Ibon Alkorta

\section{To cite this version:}

Iciar Uriarte, Aran Insausti, Emilio Cocinero, Atef Jabri, Isabelle Kleiner, et al.. Competing Dispersive Interactions: From Small Energy Differences to Large Structural Effects in Methyl Jasmonate and Zingerone. Journal of Physical Chemistry Letters, 2018, 9 (20), pp.5906-5914. 10.1021/acs.jpclett.8b02339 . hal-02330395

\section{HAL Id: hal-02330395 https://hal.science/hal-02330395}

Submitted on 23 Sep 2021

HAL is a multi-disciplinary open access archive for the deposit and dissemination of scientific research documents, whether they are published or not. The documents may come from teaching and research institutions in France or abroad, or from public or private research centers.
L'archive ouverte pluridisciplinaire HAL, est destinée au dépôt et à la diffusion de documents scientifiques de niveau recherche, publiés ou non, émanant des établissements d'enseignement et de recherche français ou étrangers, des laboratoires publics ou privés. 


\title{
Competing Dispersive Interactions: From Small Energy Diff erences to Large Structural Eff ects in Methyl Jasmonate and Zingerone
}

\author{
Iciar Uriarte,\| Aran Insausti, and Emilio J. Cocinero* \\ Departamento de Química Física, Facultad de Ciencia y Tecnología, Universidad del País Vasco (UPV/EHU), Barrio Sarriena, \\ 48940 Leioa, Spain \\ Biofisika Institute (CSIC, UPV/EHU), Universidad del País Vasco (UPV/EHU), Apartado 644, E-48080 Bilbao, Spain
}

\section{Atef Jabri $\|$ and Isabelle Kleiner*}

Laboratoire Interuniversitaire des Systéns Atmosphérques CNRS/IPSL UMR 7583, UniversitéParis-Est et Paris Diderot, 61

Avenue de General De Gaulle 94010 Creteil, France

\section{Halima Mouhib}

Laboratoire Modelisation et Simulation Multi Echelle MSME UMR 8208 CNRS, UniversitéParis-Est, 5 Boulevard Descartes, 77454 Marne-La-Valleé, France

\section{Ibon Alkortå}

Centro de Química Orgáica "Lora Tamayo", Instituto de Química Méida (IQM-CSIC), Juan de la Cierva, 3, 28006 Madrid, Spain

J. Phys. Chem. Lett. 2018, 9, 5906-5914 DOI: 10.1021/acs.jpclett.8b02339

ABSTRACT: Modern structural studies of biologically relevant molecules require an exhaustive interplay between experiment and theory. In this work, we present two examples where a poor choice of the theoretical method led to a misinterpretation of experimental results. We do that by performing a rotational spectroscopy study on two large and flexible biomolecules: methyl jasmonate and zingerone. The results show the enormous potential of rotational spectroscopy as a benchmark to evaluate the performance of theoretical methods.

I

$\mathrm{n}$ the past decade, structural studies on large, flexible organic molecules of biological interest moved into the focus of interest. The three-dimensional conformation that a biomolecule adopts is intimately related to its function in the biological medium. Hence, a lot of eff ort is devoted to the structural determination of biomolecules of increasing size. Structural studies carried out in condensed media can provide an environment comparable to biological surroundings, but the solvent or crystal packing forces may mask some key interactions. High-resolution structural studies in a supersonic jet benefit from an interaction-free environment where biomolecules can be probed without any kind of interference. The intrinsic properties of the molecule under study can be analyzed and separated from the effects arising from solvent or crystal packing. Regardless of the high-resolution spectroscopic technique (laser spectroscopy, microwave, etc..), the general strategy in these studies employs an interplay between experimental results and theoretical calculations (molecular mechanics, molecular dynamics, and quantum chemistry). This symbiosis between theory and experiment is nowadays a cornerstone in structural studies. Because of the increasing size and degrees of freedom of the molecular systems targeted, there is a need for precise theoretical models that support the interpretation of experimental results. On the other hand, theoretical methods still require a strong and reliable experimental counterpart to safeguard the results of any implemented model. Especially the combination of rotational spectroscopy with highly accurate quantum chemistry is a 
Scheme 1. Chemical Structures of Methyl Jasmonate and Zingerone
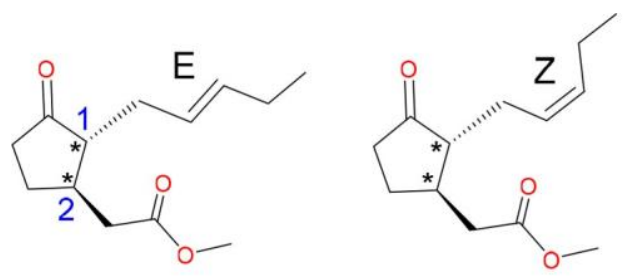

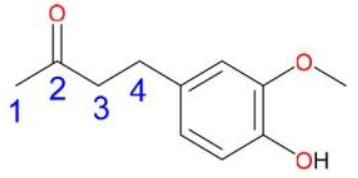

Zingerone powerful tool to study the molecular structures of large and flexible organic molecules. The efficiency of this strategy was previously shown in biologically relevant cases such as amino acids, ${ }^{1,2}$ sugars,${ }^{3-6}$ nucleobases, ${ }^{7-9}$ odorants, ${ }^{10-12}$ and some drugs. ${ }^{13-15}$

Recently, the unprecedented speed of rotational spectra acquisition obtained with broadband chirped-pulse Fourier transform microwave (CP-FTMW) spectrometers has opened new perspectives in structural chemistry. ${ }^{16-20}$ Hence, it is possible to study larger molecular systems (15-25 heavy atoms) such as biomolecules ${ }^{21}$ or large organic compounds, ${ }^{22,23}$ and their weakly bound molecular complexes. ${ }^{24-26}$ In addition, the implementation of laser vaporization sources allows one to study thermolabile systems, avoiding the undesirable thermal decomposition processes that occur when using conventional heating techniques. ${ }^{1,5}$ The experimental progress brought by CP-FTMW spectroscopy now demands the development of more accurate, faster and efficient theoretical methods to analyze and fit the experimental data.

One of the crucial points in the predictive value of the key parameters (structure, energy, dipole moments, quadrupolar tensor, ...) of larger biomolecular systems is the choice of the theoretical method. There is a large array of quantum chemical methods for that purpose. For instance, B3LYP 27,28 was historically very important, its cost-eff ectiveness making it very commonly used. However, the need to characterize weakly bound systems forced the inclusion of empirical parametriza-

tions such as Grimme's approach ${ }^{29-32}$ (D, D2, D3, D4 and its subvariants) in order to account for dispersive interactions. Minnesota functionals ${ }^{33}$ are more modern, more sophisticated, and highly parametrized, and Møller-Plesset perturbation theory ${ }^{34}$ is very reasonable for small-to-medium systems but not without limitations. Hence, the choice of method is usually subject to a trade-off between accuracy and computational cost. Systems with only covalent interactions or relatively

strong hydrogen bonds (e.g., $\mathrm{O}-\mathrm{H} \cdots \mathrm{O}, \mathrm{O}-\mathrm{H} \cdots \mathrm{N}, \mathrm{N}-\mathrm{H} \cdots \mathrm{O}$,

$15-25 \mathrm{~kJ}$

$\mathrm{mol}^{-1}$ ) can be described at almost any reasonable level of theory. However, systems stabilized by $\mathrm{O}-\mathrm{H} \cdots$ halogen, $\mathrm{C}-\mathrm{H} \cdots \mathrm{O}, \mathrm{C}-$ $\mathrm{H} \cdots \mathrm{N}, \mathrm{C}-\mathrm{H} \cdots \pi$, halogen - halogen, $\pi \cdots \pi$, lone-pair $\cdots \pi$, van der Waals, or London dispersive interactions require specific approaches to be modeled properly. In many cases, the choice of the theoretical method is done routinely without taking into account the key interactions of the system. In addition, it is wellknown that some methods are able to describe very well some weak interactions but fail at characterizing others. ${ }^{35,36}$

In the present study, we demonstrate that the choice of the appropriate quantum chemical method can be vital in the structural determination of biomolecules. In order to do so, we use the case studies of methyl jasmonate and zingerone, two of the main flavor components of jasmine and ginger, respectively (see Scheme 1 for chemical formulas). ${ }^{37,38}$ The results reported in this work clearly demonstrate the importance of carefully choosing the appropriate theoretical method for the molecular system under study. We can anticipate that a poor choice of the theoretical method may compromise the interpretation of the experimental results.

Both substances are widely present in plants. Methyl jasmonate is a vital lipidic phytohormone produced by many plants in response to biotic stress (e.g., pathogens or pests) and abiotic stress (e.g., heat, cold, wind, precipitation). It is an important signaling molecule and plays a crucial role in the communication of plants. ${ }^{39,40}$ Zingerone is the main component of ginger and it is responsible for its pungent scent. It has been shown to exhibit diuretic and neuroprotective functions, which are still not fully understood at a molecular scale. ${ }^{41}$ Besides their interesting biological effects, these molecules represent limiting cases with respect to their size and molecular weights, for both the theoretical and the experimental studies presented in this work.

Case Study 1: Methyl Jasmonate. Methyl jasmonate (MJ) consists of a cyclopentanone ring with two large flexible side chains (see Scheme 1). There are two chiral centers in the molecule, meaning that there are two diastereoisomers to consider ( $R R$ and $R S$ ). The corresponding enantiomers ( $S S$ and $S R$ ) would be equivalent by symmetry and therefore, they would give rise to the same rotational spectra. Furthermore, the double bond in one of the side chains may adopt $Z$ or $E$ configurations. Therefore, there are four diff erent structural isomers to consider (RR-Z, RR-E, RS-Z, and RS-E). Each one of them may adopt different conformations arising from rotations along single bonds. Moreover, there could be attractive intramolecular interactions (such as hydrogen bonds) between the two chains. We can infer from these structural and conformational degrees of freedom that the conformational casuistry of methyl jasmonate is very broad and complex.

The first step in this study was a fast conformational search with molecular mechanics (MM), using Merck's molecular force field (MMFF). About 100 plausible structures for each system were found in an energy window of $20 \mathrm{~kJ} \mathrm{~mol}^{-1}$. Hence, $4 \times$ $\sim 100 \approx 400$ structures had to be reoptimized with more accurate theoretical calculations to predict the relevant rotational parameters (structures, energies, dipole moments, ...). At that stage, we chose the B3LYP method, a fast and reasonably accurate method, to guide the experiment and reoptimized all the structures.

In the meantime, the rotational spectrum of methyl jasmonate was recorded in the frequency region of $6-18 \mathrm{GHz}$ using the CP-FTMW spectrometer available at the University of the Basque Country (UPV/EHU), see Figure S1 in the Supporting Information. The sample was heated up to $200{ }^{\circ} \mathrm{C}$ in a customized heating nozzle in order to build up enough concentration of methyl jasmonate in the gas phase. Two set 
Table 1. Microwave Spectroscopic Results of the Most Abundant Conformers of Methyl Jasmonate (RR, Z conformation of the double bond) and Zingerone Observed in the Molecular Jet

\begin{tabular}{|c|c|c|c|c|}
\hline \multirow[b]{2}{*}{ const. $^{a}$} & \multirow[b]{2}{*}{ Units } & \multicolumn{2}{|c|}{ Methyl Jasmonate } & \multirow{2}{*}{$\begin{array}{c}\text { Zingerone } \\
\text { Conf. Z1 }\end{array}$} \\
\hline & & Conf. MJ1 & Conf. MJ2 & \\
\hline$A_{0}$ & $\mathrm{MHz}$ & $634.08213(87)$ & $562.186(74)$ & $1023.7533(22)$ \\
\hline$B_{0}$ & $\mathrm{MHz}$ & $370.027517(80)$ & $382.806(23)$ & 434.84931 (53) \\
\hline$C_{0}$ & $\mathrm{MHz}$ & $264.431520(73)$ & $260.36873(80)$ & 352.90045 (32) \\
\hline$\Delta_{J}\left(D_{J}\right)$ & $\mathrm{kHz}$ & $0.009815(62)^{b}$ & $0.355(37)^{b}$ & $0.06592(70)^{c}$ \\
\hline$\Delta_{J K}\left(D_{J K}\right)$ & $\mathrm{kHz}$ & $0.3351(21)^{b}$ & $3.55(87)^{b}$ & $0.1088(84)^{c}$ \\
\hline$\Delta_{\mathrm{K}}\left(D_{\mathrm{K}}\right)$ & $\mathrm{kHz}$ & $0.1388(16)^{b}$ & $-15.97(2.51)^{b}$ & $0^{h}$ \\
\hline$d_{J}\left(d_{1}\right)$ & $\mathrm{kHz}$ & $0^{h}$ & $0.171(19)^{b}$ & $-0.00648(55)^{c}$ \\
\hline$d_{K}\left(d_{2}\right)$ & $\mathrm{kHz}$ & $0^{h}$ & $2.76(45)^{b}$ & $-0.00173(34)^{c}$ \\
\hline$V_{3}^{d}$ & $\mathrm{~cm}^{-1}$ & $446.03(1.67)$ & $427.88(83)$ & $336.474(81)$ \\
\hline$\sigma^{e}$ & $\mathrm{kHz}$ & 17.1 & 18.7 & 22.2 \\
\hline$N_{\text {tot }}^{f}$ & & 580 & 215 & 183 \\
\hline$N_{\mathrm{A}} / N_{\mathrm{E}^{8}}$ & & $299 / 281$ & 114/101 & $105 / 78$ \\
\hline
\end{tabular}

${ }^{a_{a}}$ The microwave data set was fitted using the program XIAM.40,41 $A, B, C$ : rotational constants. $\Delta_{J}\left(D_{J}\right), \Delta_{J K}\left(D_{J K}\right), \Delta_{K}\left(D_{K}\right), \delta_{J}\left(d_{1}\right), \delta_{K}\left(d_{2}\right)$ : centrifugal distortion constants for Watson's A reduction for methyl jasmonate and (S) reduction for zingerone, $I^{r}$ representation. Errors of the experimental data given in parentheses are in units of the last digit. ${ }^{b}$ Watson's A reduction $\Delta_{J}, \Delta_{J K}, \Delta_{K}, \delta_{J}$, and $\delta_{K}$ are used for methyl jasmonate conformer 1 (Ray's kappa $=-0.43$ ) and for conformer 2 (Ray's kappa $=-0.19$ ). cWatson's $S$ reduction $D_{J}, D_{J K}, D_{K}, d_{1}$ and $d_{2}$ are used for zingerone (Ray's kappa $=-0.75) .{ }^{d}$ Barrier to internal rotation of the methoxy methyl group and carbonyl methyl group in methyl jasmonate and zingerone, respectively. ${ }^{e}$ Standard deviation of the fit. $f$ Total number of fitted lines. 8 Number of fitted A and E species; $h$ Parameters set to zero.

Table 2. Comparison of the Experimental Values of $A_{e}, B_{e}$, and $C_{e}$ with the Theoretical Values Calculated with Diff erent Theoretical Methods (Basis Set: 6-311++G(d,p)) for Conf. MJ1 (a) and Conf. MJ2 (b) of Methyl Jasmonate

\begin{tabular}{|c|c|c|c|c|c|c|c|c|c|c|c|c|}
\hline a. & Exp & B3LYP & TPSS & PBE & B2PLYP & $\begin{array}{c}\text { B3LYP- } \\
\text { D3 }\end{array}$ & $\begin{array}{c}\text { B3LYP- } \\
\text { D3BJ }\end{array}$ & $\begin{array}{c}\text { B2PLYP } \\
\text {-D3BJ }\end{array}$ & MP2 & M06-2X & B97-D3 & $\begin{array}{l}\text { B97- } \\
\text { D3BJ }\end{array}$ \\
\hline$A_{e}$ & $641.37313(87)$ & 549.8 & 550.7 & 572.6 & 600.9 & 643.1 & 644.3 & 633.9 & 642.0 & 662.8 & 637.1 & 623.3 \\
\hline \%error $A_{e}$ & & 14.3 & 14.1 & 10.7 & 6.3 & -0.3 & -0.5 & 1.2 & -0.1 & -3.3 & 0.7 & 2.8 \\
\hline$B_{e}$ & $371.505517(80)$ & 366.1 & 366.5 & 368.2 & 369.2 & 368.1 & 367.7 & 369.3 & 370.8 & 373.9 & 365.7 & 366.7 \\
\hline \%error $B_{e}$ & & 1.4 & 1.3 & 0.9 & 0.6 & 0.9 & 1.0 & 0.6 & 0.2 & -0.7 & 1.6 & 1.3 \\
\hline$C_{e}$ & $267.38152(73)$ & 245.1 & 245.1 & 250.4 & 257.4 & 265.1 & 265.7 & 264.3 & 266.7 & 269.6 & 263.1 & 260.9 \\
\hline \%error $C_{e}$ & & 8.3 & 8.3 & 6.3 & 3.7 & 0.9 & 0.6 & 1.2 & 0.3 & -0.8 & 1.6 & 2.4 \\
\hline $\begin{array}{l}\text { CPU time } \\
\text { (h)/opt } \\
\text { cycle }\end{array}$ & & 0.8 & 3.2 & 2.3 & 15.8 & 0.9 & 0.9 & 13.8 & 5.1 & 1.0 & 1.7 & 0.9 \\
\hline
\end{tabular}

\begin{tabular}{|c|c|c|c|c|c|c|c|c|c|c|c|c|}
\hline b. & Exp & B3LYP & TPSS & PBE & B2PLYP & $\begin{array}{c}\text { B3LYP- } \\
\text { D3 }\end{array}$ & $\begin{array}{l}\text { B3LYP- } \\
\text { D3BJ }\end{array}$ & $\begin{array}{c}\text { B2PLYP } \\
\text {-D3BJ }\end{array}$ & MP2 & M06-2X & B97-D3 & $\begin{array}{l}\text { B97- } \\
\text { D3BJ }\end{array}$ \\
\hline$A_{e}$ & $560.931(74)$ & 477.2 & 488.0 & 501.2 & 532.2 & 573.1 & 568.6 & 564.9 & 572.9 & (588.2 & 567.9 & 556.6 \\
\hline$\%$ error $A_{e}$ & & 14.9 & 13.0 & 10.6 & 5.1 & -2.2 & -1.4 & -0.7 & -2.1 & -4.9 & -1.2 & 0.8 \\
\hline$B_{e}$ & $388.143(23)$ & 382.5 & 381.2 & 382.0 & 382.4 & 380.0 & 380.9 & 381.6 & 382.5 & 382.5 & 378.0 & 379.3 \\
\hline \%error $B_{e}$ & & 1.4 & 1.8 & 1.6 & 1.5 & 2.1 & 1.9 & 1.7 & 1.5 & 1.5 & 2.6 & 2.3 \\
\hline$C_{e}$ & $261.00473(80)$ & 237.4 & 240.5 & 243.9 & 253.8 & 261.5 & 261.1 & 262.2 & 266.7 & 267.6 & 259.7 & 258.3 \\
\hline \%error $C_{e}$ & & 9.1 & 7.9 & 6.6 & 2.8 & -0.2 & 0.0 & -0.5 & -2.2 & -2.5 & 0.5 & 1.0 \\
\hline $\begin{array}{l}\text { CPU time } \\
\text { (h)/opt } \\
\text { cycle }\end{array}$ & & 1.0 & 2.6 & 2.0 & 16.0 & 1.0 & 1.3 & 14.8 & 5.8 & 2.2 & 0.8 & 2.0 \\
\hline
\end{tabular}

${ }^{a}$ Note that the experimental values of $B_{e}$ are calculated such that $B_{e}=B_{0}-\Delta B_{v i b}$, and the anharmonic correction $\Delta B_{v i b}$ is estimated for each conformer separately at the B97D3/def2-SVP level of theory. Values shaded in orange correspond to Conf. MJ1' (part a) and Conf. MJ2' (part b).

of rotational transitions were spotted in the spectrum. They were first fitted separately to two semirigid asymmetric rotor Hamiltonians using Watson's A reduction. ${ }^{42}$ The rotational transitions in the spectrum displayed splittings due to tunneling effects from internal rotation of a methyl top. Those splittings have to be accounted for in the fit by appropriate 


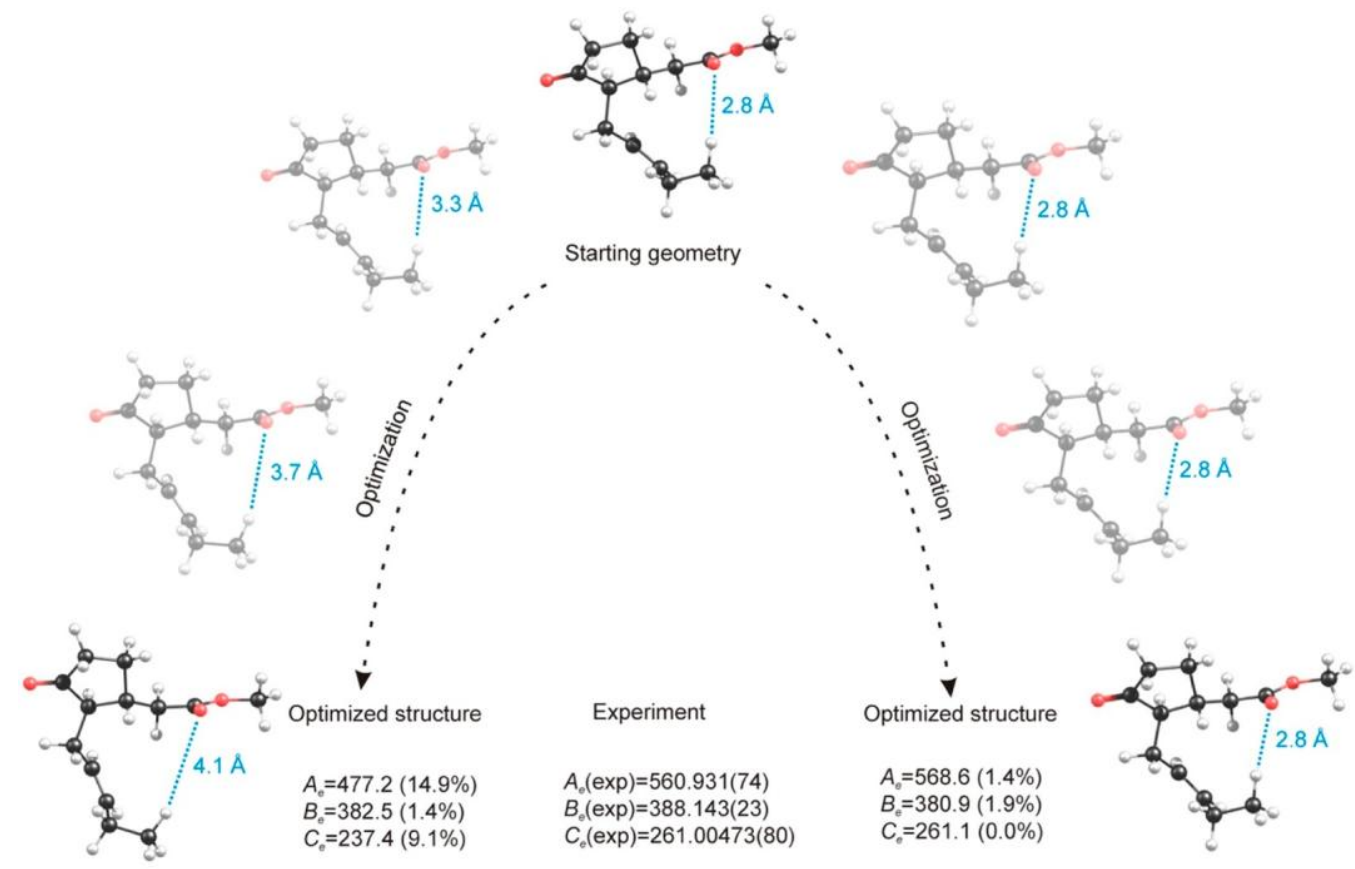

B3LYP

B3LYP-D3BJ

Conf. MJ2'

Conf. MJ2

Figure 1. Steps of the optimization of Conf. MJ2" and Conf. MJ2 of methyl jasmonate (RR, Z conformation of the double bond) using the B3LYP and B3LYP-D3BJ DFT methods, respectively, from the same starting geometry (basis set: 6-311++G(d,p)).

Hamiltonian, allowing for the estimation of the barrier for fragmentation resulting in decomposition products or internal internal rotation of the methyl top $\left(V_{3}\right)$. We used two diff erent publicly available software programs (XIAM ${ }^{43,44}$ and BELGI $^{45}$ ) for fitting the experimental results. A number of previous studies have shown that the XIAM code and the BELGI code are

complementary. ${ }^{46-48}$ As described in the Supporting Information (effective Hamiltonian section), the XIAM code takes into

account one torsional state at a time, and has a limited number of high order Hamiltonian terms implemented, whereas the BELGI-C1 code treats all torsional states together in one matrix and has many higher order terms. While XIAM benefits from the advantages of its ease of use and speed, in cases with rather low barriers, E species transitions are not always satisfactorily predicted because the interactions between torsional states can be large. Considering the importance of carefully deriving experimental rotational constants to compare with $a b$ initio methods, we decided to use both methods in this work. In the present case, the results were similar (see Tables S1-S3), and in the following, we will only refer to the XIAM fits, which are summarized in Table 1 . The experimental values of rotational and torsional parameters for the two conformers of MJ are presented in full in Table S1 of the Supporting Information.

The common strategy used in the microwave spectroscopy community to assign a molecular conformation to a given set of experimental rotational constants is to compare them to a predicted spectrum generated with the values arising from quantum chemical calculations. However, all attempts to assign the spectrum based on diff erent structural models of methyl jasmonate failed. This spectrum did not correspond to any of the predicted conformations for methyl jasmonate using the B3LYP method. Finally, we considered the possibility of sample 
reactions, but the considered species did not match the spectum either. By this point, we had carried out more than 1000 calculations but none of them reproduced the experiment. Obviously, the B3LYP method failed to provide accurate structures of the most abundant conformers of methyl jasmonate. Therefore, we decided to redo the optimizations of methyl jasmonate using the MP2 ab initio method, which finally led to structures that were in agreement with our experimental results. Its lowest lying energy conformers (Conf. MJ1 and Conf. MJ2) had not been detected by the B3LYP method, and these two were the structures that reproduced the experimental data. Although optimizations at the B3LYP level may be largely sufficient for average sized systems, there is a limit, when intramolecular interactions become too important and cannot be described properly by this method. Indeed, for methyl jasmonate the B3LYP method was insufficient to provide accurate structures that are consistent with the highly accurate experimental results.

Finally, we carried out numerous calculations with diff erent methods to compare their performance in reproducing experimental values. We always used the same basis set (6$311++\mathrm{G}(\mathrm{d}, \mathrm{p}))$. Theoretical methods yield the rotational constants at the bottom of the potential well (commonly known as $B_{e}$ equilibrium structure) while the experimental rotational constants refer to the structure at the vibrational

ground state $\mathrm{v}=0\left(B_{0}\right)$. We estimated $\Delta B_{\text {vib }}=B_{0}-B_{e}$ with anharmonic calculations at the B97D3/def2-SVP level of theory and subtracted it from the experimental rotational constants in order to obtain the semiempirical values of $B_{e}$. The latter are directly comparable to the rotational constants obtained from quantum chemical calculations. $^{22,49}$ The results are summarized in Table 2 for the two observed conformers of methyl jasmonate. 


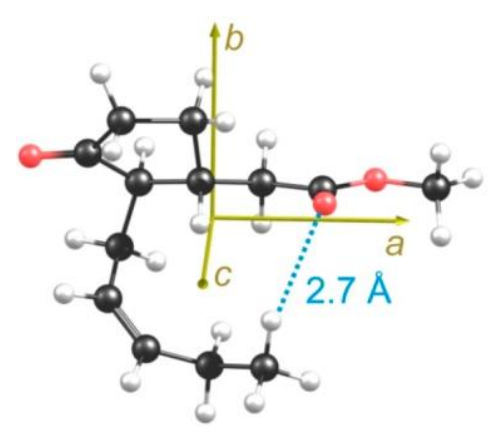

Conf. MJ1

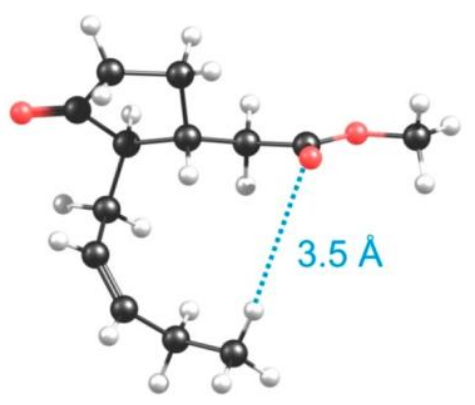

Conf. MJ1'

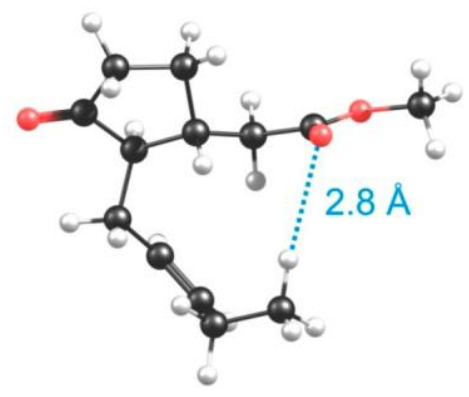

Conf. MJ2

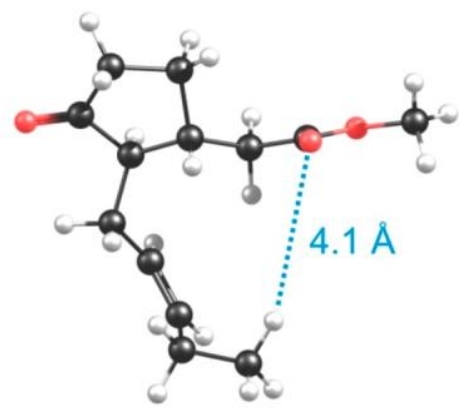

Conf. MJ2'

Figure 2. Two experimentally observed conformations of methyl jasmonate ( $R R, Z$ conformation of the double bond) (Conf. MJ1 and Conf. MJ2) as optimized at the B3LYP-D3BJ/6-311++G(d,p) level of theory, as well as the corresponding conformations Conf. MJ1' and Conf. MJ2' calculated at the B3LYP/6-311++G(d,p) level of theory. For Conf. MJ1, we also show the principal axes of inertia $a, b$, and $c$.

It is noteworthy that the B3LYP method, which does not account for dispersion effects, fails at reproducing the experimental structure (relative errors in $A$ around $15 \%$ ). This is also the case for other methods such as PBE, TPSS or B2PLYP (although B2PLYP provides significantly better results, with $\sim 6 \%$ error in the $A$ rotational constant). Only ab initio and DFT methods that considered dispersion effects (either intrinsically

or by adding a correction) were successful at reproducing experimental values. In this case, the Minnesota functional M06-

2X gives worse results (relative errors in $A$ around $4-5 \%$ ) than DFT methods corrected by Grimme's corrections (relative errors $0-2 \%$ ). Figure 1 displays the steps of the optimization of Conf. MJ 2 with the B3LYP and B3LYP-D3BJ methods starting from the same geometry. We can clearly see that with B3LYP, as the optimization progresses, the two arms move away from each other, the final distance $\mathrm{CH} \cdots$ O being $4.1 \AA$ (conformer Conf. $\mathrm{MJ} 2$ "). Hence, the $\mathrm{CH} \cdots \mathrm{O}$ interaction is not accounted for by the B3LYP method. On the contrary, using B3LYP-D3BJ, the two arms remain close to each other, held together by the $\mathrm{CH} \cdots$ O weak interaction (final distance $2.8 \AA$, Conf. MJ2). We infer that the dispersion correction D3BJ has a dramatic eff ect on the rotational constants. In particular, the $A$ rotational constant is strongly influenced by dispersion in the case of methyl jasmonate because the principal axis $a$ lies along the two side chains. Hence, the moment of inertia $I_{a}$ (to which $A$ is inversely proportional) is very sensitive to the coordinates of the atoms in the side chains (see Figure 2 for the principal axes of inertia). The eff ect of dispersion is so pronounced in this molecule that the assignment to the molecular conformations was not possible with the B3LYP methodology. 
To summarize, the two experimental conformations are assigned to Conf. MJ1 and Conf. MJ2: both of them are $R R$ diasteroisomers (or $S S$ ), the configuration of the double bond in the side chain is $Z$ and both conformations are stabilized by a $\mathrm{C}-\mathrm{H} \cdots \mathrm{O}$ weak hydrogen bond (WHB). The corresponding 3D

structures are depicted in Figure 2, along with the structures of Conf. MJ1' and Conf. MJ2', i.e., the conformations predicted from calculations where no dispersion is included.

Case Study 2: Zingerone. Zingerone (see Scheme 1) consists of an 0 -methoxyphenol moiety with a flexible chain in para position with respect to the hydroxy group. In order to guide the spectral search, we predicted the most favorable structures employing the same strategy as described above and commonly used in the

literature: we performed MM to have an initial screening of the conformational scope (14 structures were found in $15 \mathrm{~kJ}$ $\mathrm{mol}^{-1}$ ) and then we reoptimized all of them with the MP2 robust method.

Later, the rotational spectrum of zingerone in the 6-18 $\mathrm{GHz}$ frequency region was recorded using the FTMW and $\mathrm{CP}-$

FTMW spectrometers at the University of the Basque Country (UPV/EHU) see Figure S2 of the Supporting Information. The sample was heated to $200{ }^{\circ} \mathrm{C}$ in a customized heating nozzle in order to bring it into the gas phase. A set of rotational transitions corresponding to the $A$ species of one conformation of the molecule was assigned and fitted to a semirigid asymmetric rotor Hamiltonian in Watson's S-reduction. Splittings between A and E species due to the internal rotation were accounted for in the fit by appropriate operators in the Hamiltonian. The results of the fit are summarized in Table 1.

Surprisingly, there was no correspondence between experi- ment and theory. The discrepancy in the rotational constants 
was remarkable. Diff erences of $\sim 17 \%$ indicated that the predicted structure was not the experimentally observed structure, despite having used a method as reliable as MP2 theory.

In this case, we decided to reoptimize all the structures using DFT theory, in particular the B3LYP-D3BJ functional. Interestingly, the results generated with this functional were not comparable to those obtained with the MP2 method. With both methods, all the lowest lying conformations were stabilized by an $\mathrm{OH} \cdots$ O interaction between the hydroxy and the methoxy group. However, the global minimum was diff erent for the MP2 and B3LYP-D3BJ calculations. Additionally, the lowest lying structure predicted by B3LYP-D3BJ was not predicted using the MP2 method. The two conformations predicted as global minima in zingerone are shown in Figure 3. The first one (Conf.

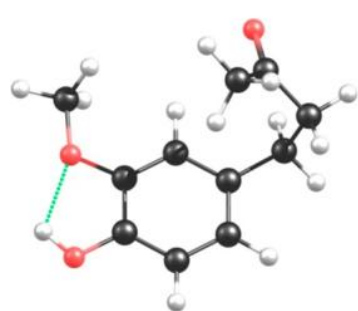

Conf. Z1

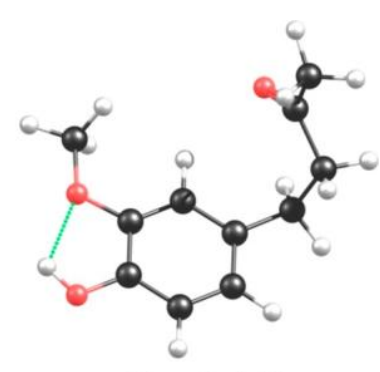

Conf. Z2
Figure 3. Conformations of zingerone predicted as global minima with the MP2 (Conf. Z1) and B3LYP-D3BJ methods (Conf. Z2), using the basis set $6-311++G(d, p)$.
Z1, MP2 structure exhibits an attractive $\mathrm{CH} \cdots \pi$ interaction between the terminal $\mathrm{CH}_{3}$ in the flexible chain and the $\pi$ cloud in the aromatic ring. This interaction forces the side chain to tilt toward the ring. It should be noted that this structure is not predicted by B3LYP (or TPSS, PBE, and B2PLYP, i.e., methods that do not account for dispersive interactions). In the second conformation (Conf. Z2, B3LYP-D3BJ structure), no such interaction occurs and the side chain is perpendicular to the ring. This conformer exhibits a $\mathrm{C} \mathrm{O} \cdot \mathrm{\cdots} \mathrm{H}-\mathrm{C}$ attractive interaction. This conformation was not predicted by the MP2 method. To summarize, we have two possible global minima, one of which was not predicted at the MP2/6-311++G(d,p) level of theory.

In addition, we extended the predictions to other theoretical models. Table 3 compiles the results of those calculations. Clearly, the result of the optimizations highly depend on the applied theoretical method. The balance between the $\mathrm{CH} \cdots \pi$ interaction (Conf. Z1) and the $\mathrm{CH} \cdots \mathrm{O}$ interaction (Conf. Z2) is very subtle. It is a challenging task for theoretical models to weigh the strength of the two interactions, since both are weak hydrogen bonds (WHB) with small stabilization energies below $4 \mathrm{kcal} \mathrm{mol}{ }^{-1} .50$ Regarding the theoretical methods, three diff erent trends are observed. They are summarized in Figure 4 with the three common methods MP2, B3LYP, and B3LYPD3BJ. We can observe that B3LYP-D3BJ is capable of reproducing both conformations if the correct starting geometry is chosen. B3LYP always yields Conf. Z2 stabilized by $\mathrm{CH} \cdots \mathrm{O}$ interaction, that is, with the lateral chain perpendicular to the plane of the aromatic ring. Finally, MP2 calculations result always in Conf. Z1 with a $\mathrm{CH} \cdots \pi$ interaction, where the chain is bent toward the benzene ring. Table 3 shows the diff erences in

Table 3. Comparison of the Experimental Values of $A_{e}, B_{e}$, and $C_{e}$ with the Theoretical Values Calculated with Diff erent Theoretical Methods (Basis Set: 6-311++G(d,p)) for Conf. Z2 (a) and Conf. Z1 (b) of Zingeronea

\begin{tabular}{|c|c|c|c|c|c|c|c|c|c|c|c|c|}
\hline a. & Exp & B3LYP & TPSS & PBE & B2PLYP & $\begin{array}{l}\text { B3LYP- } \\
\text { D3 }\end{array}$ & $\begin{array}{l}\text { B3LYP- } \\
\text { D3BJ }\end{array}$ & $\begin{array}{c}\text { B2PLYP } \\
\text {-D3BJ }\end{array}$ & MP2 & M06-2X & B97-D3 & $\begin{array}{l}\text { B97- } \\
\text { D3BJ }\end{array}$ \\
\hline$A_{e}$ & $1029.5243(22)$ & 1034.5 & 1021.8 & 1024.6 & 1021.2 & 1016.9 & 1017.8 & 1009.9 & NA* & 1028.5 & 1001.7 & NA \\
\hline \%error $A_{e}$ & & -0.5 & 0.8 & 0.5 & 0.8 & 1.2 & 1.1 & 1.9 & NA & 0.1 & 2.7 & NA \\
\hline$B_{e}$ & $441.49935(53)$ & 421.4 & 423.0 & 422.7 & 433.2 & 437.5 & 439.4 & 444.7 & NA & 441.7 & 436.9 & NA \\
\hline$\%$ error $B_{e}$ & & 4.6 & 4.2 & 4.3 & 1.9 & 0.9 & 0.5 & -0.7 & NA & -0.1 & 1.0 & NA \\
\hline$C_{e}$ & $356.47486(32)$ & 340.6 & 342.9 & 341.4 & 351.4 & 352.3 & 354.2 & 360.6 & NA & 357.6 & 351.7 & NA \\
\hline$\%$ error $C_{e}$ & & 4.5 & 3.8 & 4.2 & 1.4 & 1.2 & 0.6 & -1.1 & NA & -0.3 & 1.3 & NA \\
\hline $\begin{array}{c}\text { CPU } \\
\text { time/opt } \\
\text { cycle }\end{array}$ & & 0.4 & 0.006 & 0.5 & 4.7 & 0.8 & 0.4 & 4.7 & NA & 0.5 & 0.004 & NA \\
\hline
\end{tabular}

\begin{tabular}{c|cccccccccccc}
\cline { 2 - 12 } \multicolumn{1}{l|}{} & B3LYP & TPSS & PBE & B2PLYP & B3LYP-D3 & $\begin{array}{c}\text { B3LYP- } \\
\text { D3BJ }\end{array}$ & $\begin{array}{c}\text { B2PLYP- } \\
\text { D3BJ }\end{array}$ & MP2 & M06-2X & B97-D3 & B97-D3BJ \\
\hline \hline$A_{e}$ & NA* & NA & NA & NA & 991.7 & 994.6 & 994.2 & 994.7 & 999.8 & 985.5 & 987.7 \\
$B_{e}$ & NA & NA & NA & NA & 483.6 & 487.1 & 488.9 & 509.3 & 503.5 & 482.4 & 486.5 \\
$C_{e}$ & NA & NA & NA & NA & 390.4 & 395.5 & 398.2 & 413.6 & 407.5 & 390.2 & 395.0 \\
$\begin{array}{c}\text { CPU } \\
\text { time } / \text { opt } \\
\text { cycle }\end{array}$ & NA & NA & NA & NA & 0.6 & 1.4 & 7.5 & 2.5 & 1.9 & 0.6 & 0.006 \\
\hline
\end{tabular}

${ }^{a}$ Note that the experimental values of $B_{e}$ are calculated such that $B_{e}=B_{0}-\Delta B_{v i b}$, and the anharmonic correction $\Delta B_{v i b}$ is estimated for each conformer separately at the B97D3/def2-SVP level of theory. NA = Not Applicable, the optimization leads either to Conf. Z1 (part a) or Conf Z2 (part b). 
the rotational constants. Again, the choice of the theoretical method is crucial to success. The rotational constants are very sensitive to both conformers and we will only be able to discern unambiguously the structure of zingerone through microwave spectroscopy.

By comparing the experimental values of the rotational constants with the theoretical predictions, we can conclude that the experimental conformation of zingerone is Conf. Z2, which is stabilized by a $\mathrm{CH} \cdots \mathrm{O}$ interaction. This means that MP2/ 6 $311++\mathrm{G}(\mathrm{d}, \mathrm{p})$, which is a very commonly used and most trusted method in the rotational spectroscopy community, fails to predict the experimentally observed conformation of zingerone as minimum energy structure. In fact, the observed conformer is not even predicted by this method. MP2 overestimates the $\mathrm{CH} \cdots \pi$ interaction and, as the balance between WHBs is so subtle, the real structure is not predicted by the MP2 method. In order to visualize the subtlety of the competition between the two WHBs, we calculate the barrier between the two structures by performing a relaxed scan of the $1-2-3-4$ dihedral angle (see Scheme 1 for atom numbering). Moreover, we perform this scan using two different methods (M06-2X and B3LYP$\mathrm{D} 3 \mathrm{BJ})$

to evaluate the response of both of them in the WHBs competition. Figure 5 displays the two scans. We can observe the local minima corresponding to Conf. Z1 and Conf. Z2 in both scans. It is worth noting that these minima are almost isoenergetic. The energy diff erence between both of them is

less than $0.5 \mathrm{~kJ} \mathrm{~mol}^{-1}$ (this value is probably within the error of the theoretical calculation). Moreover, M06-2X and B3LYP$\mathrm{D} 3 \mathrm{BJ}$ predict that the barrier between the two structures is very low $\left(\sim 1 \mathrm{~kJ} \mathrm{~mol}^{-1}\right)$, meaning that it can be easily overcome in the supersonic expansion and therefore, the system would relax to the most stable conformation. This behavior has been previously observed in rotational spectra and is well reported in the literature. ${ }^{51,52}$ The most relevant difference between the two

theoretical methods is precisely the energetic order of the conformers. While M06-2X predicts that the most stable conformation is Conf. Z1 (CH $\cdots \pi$ interaction), B3LYP-D3BJ predicts Conf. $\mathrm{Z} 2$ as the most stable structure $(\mathrm{CH} \cdots \mathrm{O}$ interaction). The small energy diff erence and the low barrier between the two conformations proves the subtlety of the competition between the two interactions, with different theoretical methods tipping the balance in favor of one structure or the other.

\section{Starting geometries}

\section{ptimized structures}

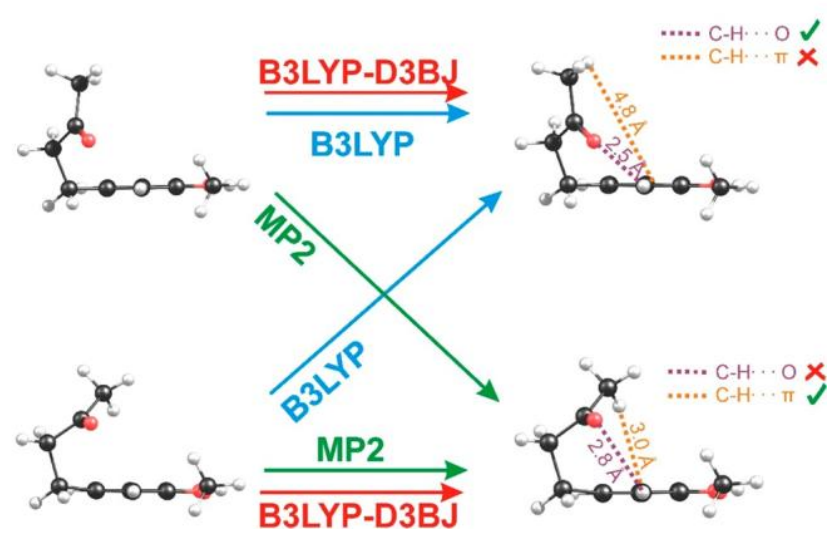

a.

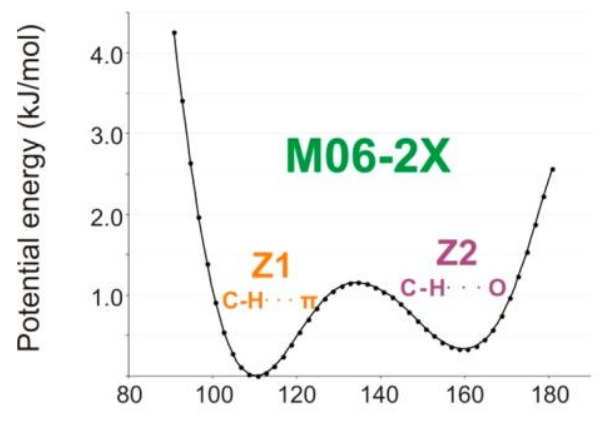

Dihedral angle 1-2-3-4 (degrees)

b.

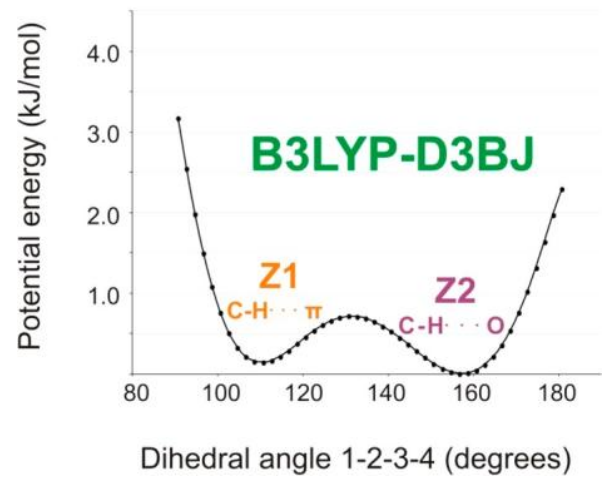

Figure 5. Potential energy scans of the 1-2-3-4 dihedral angle of zingerone to evaluate the energy barrier between Conf. Z1 (stabilized by a $\mathrm{C}-\mathrm{H} \cdots \mathrm{T}$ interaction) and Conf. $\mathrm{Z} 2$ (stabilized by a $\mathrm{C}-\mathrm{H} \cdots \mathrm{O}$ interaction). Two different theoretical methods have been used: M06-

2X (a) and B3LYP-D3BJ (b), in order to evaluate their behavior with respect to the zingerone molecular system. The basis set is 6-311+ $+\mathrm{G}(\mathrm{d}, \mathrm{p})$.

Figure 4. Schematic representation of the prototype behavior of theoretical methods in the conformational casuistry of zingerone. 
This study demonstrates that rotational spectroscopy combined with theoretical methods is a powerful strategy, which can be broadly applied to flexible organic compounds. However, one must be cautious in the use of different theoretical methods. There is currently no strategy to avoid brute force optimizations in this kind of molecular systems (for example, force fields are poorly parametrized for the accurate description of averaged sized biologically active ligands). Therefore, benchmark studies such as the ones presented here are extremely necessary. We can infer from the studies on methyl jasmonate and zingerone that using several distinct levels of theory allows one to get a good Overview of the conformational

\section{ACKNOWLEDGMENTS}

A.J., H.M., and I.K. would like to acknowledge the CNRS InFinity program and the OSU/Efluve for funding; H.M. thanks the European COST initiative MOLIM for a STSM fellowship; E.J.C. and I.U. thank the MINECO (CTQ2017-89150-R) and the UPV/EHU (UFI11/23 and PPG17/10) for funds. Computational resources and laser facilities of the UPV-EHU were used in this work (SGIker). Computational resources of the MSME laboratory of the University Paris-Est were used in this work.

\section{REFERENCES}

(1) Lesarri, A.; Mata, S.; Cocinero, E. J.; Blanco, S.; Lópz, J. C.; Alonso, J. L. The Structure of Neutral Proline. Angew. Chem., Int. Ed. 2002, 41, 4673-4676.

(2) Lesarri, A.; Sánhez, R.; Cocinero, E. J.; Lópz, J. C.; Alonso, J. L. Coded Amino Acids in Gas Phase: The Shape of Isoleucine. J. Am. Chem. Soc. 2005, 127, 12952-12956.

(3) Cocinero, E. J.; Carcabbal, P.; Vaden, T. D.; Simons, J. P.; Davis, B. G.Sensing the Anomeric Effect in a Solvent-Free Environment. Nature 2011, 469, 76-80.

(4) Cocinero, E. J.; Gamblin, D. P.; Davis, B. G.; Simons, J. P. The Building Blocks of Cellulose: The Intrinsic Conformational Structures of Cellobiose, Its Epimer, Lactose, and Their Singly Hydrated Complexes. J. Am. Chem. Soc. 2009, 131,11117-11123.

(5) Cocinero, E. J.; Lesarri, A.; Ecija, P.; Basterretxea, F. J.; Grabow, J. U.; Fernádez, J. A.; Castaño, F. Ribose Found in the Gas Phase. Angew. Chem., Int. Ed. 2012, 51, 3119-3124.

(6) Ecija, P.; Uriarte, I.; Spada, L.; Davis, B. G.; Caminati, W.; Basterretxea, F. J.; Lesarri, A.; Cocinero, E. J. Furanosic Forms of Sugars: Conformational Equilibrium of Methyl $\beta$-D-Ribofuranoside. Chem. Commun. 2016, 52, 6241-6244.

(7) Nir, E.; Kleinermanns, K.; De Vries, M. S. Pairing of Isolated Nucleic-Acid Bases in the Absence of theDNABackbone. Nature 2000, 408, 949-951.

(8) Alonso, J. L.; Peña, I.; Lópz, J. C.; Vaquero, V. Rotational Spectral Signatures of Four Tautomers of Guanine. Angew. Chem., Int. Ed. 2009, $48,6141-6143$.

(9) Favero, L. B.; Uriarte, I.; Spada, L.; Ecija, P.; Calabrese, C.; Caminati, W.; Cocinero, E. J. Solving the Tautomeric Equilibrium of Purine through Analysis of the Complex Hyperfine Structure of the

Four ${ }^{14} \mathrm{~N}$ Nuclei. J. Phys. Chem. Lett. 2016, 7, 1187-1191.

(10) Mouhib, H.; Stahl, W.; Lüthy, M.; Büchel, M.; Kraft, P. Cassis Odor through Microwave Eyes: Olfactory Properties and Gas-Phase Structures of All the Cassyrane Stereoisomers and Its Dihydro Derivatives. Angew. Chem., Int. Ed. 2011, 50, 5576-5580.

(11) Nguyen, H. V. L.; Mouhib, H.; Klahm, S.; Stahl, W.; Kleiner, I. A Touch of Lavender: Gas-Phase Structure and Dynamics of the Monoterpene Linalool Validated by Microwave Spectroscopy. Phys. Chem. Chem. Phys. 2013, 15, 10012-10018.,

(12) Loru, D.; Quesada-Moreno, M. M.; Aviles-Moreno, J. R.; Jarman, N.; Huet, T. R.; Lopz-Gonzakz, J. J.; Sanz, M. E. Conformational landscape. This work demonstrates that microwave spectroscopy can benchmark new theoretical models very much in need to guide the experiments.

\section{ASSOCIATED CONTENT}

\section{Supporting Information}

The Supporting Information is available free of charge on the ACS Publications website at DOI: 10.1021/acs.jpclett.8b02339.

Experimental and computational details, rotational spectra of methyl jasmonate and zingerone, experimental spectroscopic parameters of all observed species

of experimental rotational transitions (PDF)

Flexibility of Limonene Oxide Studied By Microwave Spectroscopy. ChemPhysChem 2017, 18, 274-280.

(13) LeonI.; MillanJ.; Cocinero, E. J.; Lesarri, A.; Fernadez, J. A. Water Encapsulation by Nanomicelles. Angew. Chem., Int. Ed. 2014, 53, $12480-12483$.

(14) LeónI.; MillánJ.; Cocinero, E. J.; Lesarri, A.; Fernádez, J. A. Shaping Micelles: The Interplay between Hydrogen Bonds and Dispersive Interactions. Angew. Chem., Int. Ed.2013,52,7772-7775.

(15) Lesarri, A.; Vega-Toribio, A.; Suenram, R. D.; Brugh, D. J.; Grabow, J. U. The Conformational Landscape of the Volatile Anesthetic Sevoflurane. Phys. Chem. Chem. Phys. 2010, 12, 9624-9631.

(16) Brown, G. G.; Dian, B. C.; Douglass, K. O.; Geyer, S. M.; Shipman, S. T.; Pate, B. H. A Broadband Fourier Transform Microwave Spectrometer Based on Chirped Pulse Excitation. Rev. Sci.Instrum. 2008, 79, 053103.

(17) Shipman, S. T.; Pate, B. H. New Techniques in Microwave Spectroscopy. Handbook of High-resolution spectroscopy 2011, 801827.

(18) Neill, J. L.; Shipman, S. T.; Alvarez-Valtierra, L.; Lesarri, A.; Kisiel, Z.; Pate, B. H. Rotational Spectroscopy of Iodobenzene and Iodobenzene-Neon with a Direct Digital 2-8 GHz Chirped-Pulse Fourier Transform Microwave Spectrometer. J. Mol. Spectrosc. 2011, 269, 21-29.

(19) Perez, C.; Muckle, M. T.; Zaleski, D. P.; Seifert, N. A.; Temelso, B.; Shields, G. C.; Kisiel, Z.; Pate, B. H. Structures of Cage, Prism, and Book Isomers of Water Hexamer from Broadband Rotational Spectrosçopy. Science 2012, 336, 897-901.

(20) Perez, C.; Lobsiger, S.; Seifert, N. A.; Zaleski, D. P.; Temelso, B.; Shields, G. C.; Kisiel, Z.; Pate, B. H. Broadband Fourier Transform Rotational Spectroscopy for Structure Determination: The Water Heptamer. Chem. Phys. Lett. 2013, 571, 1-15.

(21) Uriarte, I.; Perez, C.; Caballero-Mancebo, E.; Basterretxea, F. J.; Lesarri, A.; Fernadez, J. A.; Cocinero, E. J. Structural Studies of Nicotinoids: Cotinine versus Nicotine. Chem. - Eur. J. 2017, 23, 72387244.

(22) Fokin, A. A.; Zhuk, T. S.; Blomeyer, S.; Perez, C.; Chernish, L. V.; Pashenko, A.E.; Antony,J.;Vishnevskiy, Y.V.;Berger, R.J.F.; Grimme, S.; et al. Intramolecular London Dispersion Interaction Effects on GasPhase and Solid-State Structures of Diamondoid Dimers. J. Am. Chem. Soc. 2017, 139, 16696-16707.

(23) Domingos, S. R.; Cnossen, A.; Buma, W. J.; Browne, W. R.; Feringa, B. L.; Schnell, M. Cold Snapshot of a Molecular Rotary Motor Captured by High-Resolution Rotational Spectroscopy. Angew. Chem., Int. Ed. 2017, 56, 11209-11212.

(24) Seifert, N. A.; Zaleski, D. P.; Pérez, C.; Neill, J. L.; Pate, B. H.; Vallejo-Lõpez, M.; Lesarri, A.; Cocinero, E. J.; Castaño, F.; Kleiner, I. Probing the $\mathrm{C}-\mathrm{H} \cdots \pi$ Weak Hydrogen Bond in Anesthetic Binding: The Sevoflurane-Benzene Cluster. Angew. Chem., Int. Ed. 2014, 53, 32103213.

(25) Steber, A. L.; Perez, C.; Temelso, B.; Shields, G. C.; Rijs, A. M.; Pate, B. H.; Kisiel, Z.; Schnell, M. Capturing the Elusive Water Trimer from the Stepwise Growth of Water on the Surface of the Polycyclic Aromatic Hydrocarbon Acenaphthene. J. Phys. Chem. Lett. 2017, 8, 5744-5750.

(26) Ghosh, S.; Thomas, J.; Huang, W.; Xu, Y.; Jägr, W. Rotational Spectra of Two Hydrogen-Bonded Methyl Salicylate Monohydrates: Relative Stability and Tunneling Motions. J. Phys. Chem. Lett. 2015, 6, 3126-3131.

(27) Becke, A.D.Density-Functional Thermochemistry.III.The Role 
of Exact Exchange. J. Chem. Phys. 1993, 98, 5648- 5652.

(28) Lee, C.; Yang, W.; Parr, R. G. Development of the Colle-Salvetti

Correlation-Energy Formula into a Functional of the Electron

Density.

Phys. Rev. B: Condens. Matter Mater. Phys. 1988, 37, 785-789.

(29) Grimme, S. Semiempirical GGA-Type Density Functional

Constructed with a Long-Range Dispersion Correction. J.

Comput. Chem. 2006, 27, 1787-1799.

(30) Grimme, S. Accurate Description of van Der Waals Complexes by Density Functional Theory Including Empirical Corrections.

J. Comput. Chem. 2004, 25, 1463-1473. 
(31) Grimme, S.; Ehrlich, S.; Goerigk, L. Effect of the Damping Function in Dispersion Corrected Density Functional Theory. J. Comput. Chem. 2011, 32, 1456-1465.

(32) Caldeweyher, E.; Bannwarth, C.; Grimme, S. Extension of the D3 Dispersion Coefficient Model. J. Chem. Phys. 2017, 147, 034112.

(33) Zhao, Y.; Truhlar, D. G. The M06 Suite of Density Functionals for Main Group Thermochemistry, Thermochemical Kinetics, Noncovalent Interactions, Excited States, and Transition Elements: Two New Functionals and Systematic Testing of Four M06-Class Functionals and 12 Other Function. Theor. Chem. Acc. 2008, 120, 215-241.

(34) Møller, C.; Plesset, M. S. Note on an Approximation Treatment for Many-Electron Systems. Phys. Rev. 1934, 46, 618-622.

(35) Christensen, A. S.; Kubar,T.; Cui, Q.; Elstner, M. Semiempirical Quantum Mechanical Methods for Noncovalent Interactions for Chemical and Biochemical Applications. Chem. Rev. 2016, 116, $5301-5337$.

(36) Brandenburg, J. G.; Hochheim, M.; Bredow, T.; Grimme, S. LowCost Quantum Chemical Methods for Noncovalent Interactions. J.

Phys. Chem. Lett. 2014, 5, 4275-4284.

(37) Acree, T. E.; Nishida, R.; Fukami, H. Odor Thresholds of the Stereoisomers of Methyl Jasmonate. J. Agric. Food Chem. 1985, 33, $425-427$.

(38) Asamitsu, Y.; Nakamura, Y.; Ueda, M.; Kuwahara, S.; Kiyota, H. Synthesis and Odor Description of Both Enantiomers of Methyl 4,5Didehydrojasmonate, a Component of Jasmin Absolute. Chem.

Biodiversity 2006, 3, 654-659.

(39) Cheong, J. J.; Choi, Y. Do. Methyl Jasmonate as a Vital Substance in Plants. Trends Genet. 2003, 19, 409-413.

(40) Avanci, N. C.; Luche, D. D.; Goldman, G. H.; Goldman, M. H. Jasmonates Are Phytohormones with Multiple Functions, Including Plant Defense and Reproduction. GMR, Genet. Mol. Res. 2010, 9, 484505.

(41) Fay, H. A. C. A Highly Effective and Selective Male Lure for Bactrocera Jarvisi (Tryon) (Diptera: Tephritidae). Aust. J. Entomol.

2012, 51, 189-197.

(42) Watson, J. K. G. Vibrational Spectra and Structure; During, J. R., Ed.; Elsevier: Amsterdam, 1977.

(43) Hartwig, H.; Dreizler, H. The Microwave Spectrum of Trans-2,3Dimethyloxirane in Torsional Excited States. Z. Naturforsch., A: Phys.

Sci. 1996, 51 (8), 923-932.

(44) Hansen, N.; Mädr, H.; Bruhn, T. A Molecular Beam Fourier Transform Microwave Study of O-Tolunitrile: ${ }^{14} \mathrm{~N}$ Nuclear Quadrupole Coupling and Methyl Internal Rotation Effects. Mol. Phys. 1999, 97, 587.

(45) Kleiner, I.; Hougen, J. T. Rho-Axis-Method Hamiltonian for Molecules Having One Methyl Rotor and C1 Point-Group Symmetry at Equilibrium. J. Chem. Phys. 2003, 119, 5505-5509.

(46) Nguyen, H. V. L.; Jabri, A.; Van, V.; Stahl, W. Methyl Internal Rotation in the Microwave Spectrum of Vinyl Acetate. J. Phys. Chem. A 2014, 118, 12130-12136.

(47) Eibl, K.; Kannengießer, R.; Stahl, W.; Nguyen, H. V. L.; Kleiner, I. Low Barrier Methyl Rotation in 3-Pentyn-1-ol as Observed by Microwave Spectroscopy. Mol. Phys. 2016, 114, 3483-3489.

(48) Ferres, L.; Stahl, W.; Kleiner, I.; Nguyen, H. V. L. The Effect of Internal Rotation in P-Methyl Anisole Studied by Microwave Spectroscopy. J. Mol. Spectrosc. 2018, 343, 44-49.

(49) Vogt, N.; Demaison, J.; Cocinero, E. J.; Ecija, P.; Lesarri, A.; Rudolph, H. D.; Vogt, J. The Equilibrium Molecular Structures of 2Deoxyribose and Fructose by the Semiexperimental Mixed Estimation Method and Coupled-Cluster Computations. Phys. Chem. Chem. Phys. 2016, 18, 15555-15563.

(50) Desiraju, G. R.; Steiner, T. The Weak Hydrogen Bond in Structural

Chemistry and Biology; Oxford Univ. Press: Oxford, U.K., 1999.

(51) Ruoff, R. S.; Klots, T. D.; Emilsson, T.; Gutowsky, H. S. Relaxation of Conformers and Isomers in Seeded Supersonic Jets of Inert Gases. J. Chem. Phys. 1990, 93, 3142-3150.

(52) Godfrey, P. D.; Brown, R. D.; Rodgers, F. M. The Missing Conformers of Glycine and Alanine: Relaxation in Seeded Supersonic Jets. J. Mol. Struct. 1996, 376, 65-81. 